\title{
Simultaneous biodegradation of dimethyl sulfide and 1-propanethiol by Pseudomonas putida S-1 and Alcaligenes sp. SY1: "Lag”" cause, reduction and kinetics exploration
}

Qian Li

Zhejiang Ocean University

Zeqin Tang

Zhejiang University of Technology

Jiahui Zhang

Zhejiang Ocean University

Jingtao Hu

Zhejiang University of Technology

Jianmeng Chen

Zhejiang Ocean University

DongZhi Chen ( $\square$ chendz@zjou.edu.cn )

Zhejiang Ocean University

\section{Research Article}

Keywords: Simultaneous biodegradation, 1-propanethiol, Dimethyl sulfide, Lag reduction, Kinetics

Posted Date: November 30th, 2021

DOI: https://doi.org/10.21203/rs.3.rs-1055406/v1

License: (c) (i) This work is licensed under a Creative Commons Attribution 4.0 International License.

Read Full License 


\section{Abstract}

Simultaneous biodegradation of malodorous 1-propanethiol (PT) and dimethyl sulfide (DMS) inoculated with Pseudomonas putida S-1 and Alcaligenes sp. SY1 were investigated and interactions implicated were explored. Results showed that PT was completely degraded in $33 \mathrm{~h}$, while a lag of $10 \mathrm{~h}$ was observed for DMS degradation alone, and the lag even extended to $81 \mathrm{~h}$ in the binary mixture. Mechanism analysis found that the lag was mainly attributed to the exposure of DMS degrader (Alcaligenes sp. SY1), rather than PT metabolites and PT degrader. The exposure time and PT concentration influenced the lag duration much. Citric acid could effectively reduce the lag. Pseudo first-order model was proved suitable for the description of PT degradation, revealing that PT degradation could be enhanced in presence of DMS regardless of its concentration. A modified Gompertz model, incorporated the lag phase, was developed for the description of DMS degradation in the mixture, revealing that DMS degradation depended on the initial PT concentration. When the lag was not considered, PT with low-concentration

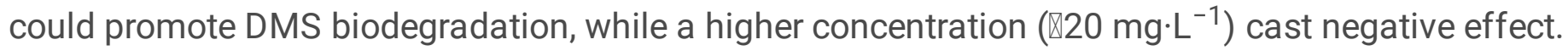

\section{Introduction}

Volatile organic compounds (VOCs) are widely considered as major precursors for the photochemical smog and haze, imposing great threats on environment quality and human health. As a typical kind of VOCs, volatile organic sulfur compounds (VOSCs) are highly toxic and malodorous with extremely low smell threshold, (Padhi and Gokhale 2017). They are not only the main composition of traditional malodor sources, i.e. waste water treatment plants, waste landfills, livestock and poultry farm etc., but also closely related with pharmaceutical chemicals, petroleum refineries, papermaking and other industries (Giri and Pandey 2013; Giri et al. 2014). So far, many treatment technologies, like condensation, UV oxidation, regenerative thermal oxidization, catalytically incineration, adsorption etc., have been employed for the disposal of odorous and hazardous organic compounds (Durme et al. 2008; Zhang et al. 2017). Thereinto, biotechnology is one of the most applicable ways for the purification of VOSCs, due to its various advantages of ecological, low cost and no secondary pollution etc., especially for the waste gas with high-volume and low-concentration (Jo and Shin 2010; Qiu and Deshusses 2017; Kennes and Veiga 2013).

To date, numerous microorganisms have been acclimated and isolated for the degradation of VOSCs. These microorganisms are usually highly specific, only one or one kind of VOSC compounds can be efficiently degraded. However, multiple waste gases usually coexist in practical situations. Unknown interactions are likely to occur during their degradation processes, which may largely influence their original degradation behaviors. It was reported that the removal of dimethyl sulfide (DMS) and dimethyl disulfide were affected by the presence of methyl mercaptan and $\mathrm{H}_{2} \mathrm{~S}$ (Wani, Lau, and Branion 1999; Li et al. 2003). In addition, substrate interactions, including co-metabolism, sequential degradation, competitive inhibition, uncompetitive inhibition, and noncompetitive inhibition, were also observed in many studies (Chen et al. 2008; Chen et al. 2007; Chen et al. 2018; Zhou et al. 2011). Chignell et al. claimed that an antagonistic interaction existed between P. putida KT2440 and Bacillus atrophaeus 1942 
when co-cultured on agar plates (Chignell et al. 2018). To be noted, negative effects (inhibition), rather than positive effects (enhancement), were more frequently reported when several kinds of gases were simultaneously degraded (Zhou, Huang, and Shen 2016; Bielefeldt and Cort 2005).

DMS and 1-propanethiol (PT) are two typical malodorous VOSCs in industrial parks (Han et al. 2018; Ras, Borrull, and Marcé 2008). However, researches on their simultaneous removal were very limited. Recently, a solid composite microbial inoculant composed of Alcaligenes sp. SY1 and Pseudomonas putida S1 was successfully prepared, which showed improved removal performance and shortened start-up period, when inoculated in biotrickling filters (Chen et al. 2018). Nevertheless, the specific interactions involved in their degradation process were untested, remaining to be elucidated.

Degradation kinetic study is an important analytical method to unveil the mechanism involved substrate interactions. The Monod model is usually used in investigating the non-inhibitory biomass growth of a pure culture with restricted substrate (Pala-Ozkok et al. 2012), and the effects of substrate inhibition on biomass growth at high concentrations have been described by a special model based on Monod or another model. For example, the sum kinetics with interaction parameter (SKIP) incorporating the interaction factor $\mathrm{I}_{\mathrm{i}, \mathrm{j}}$, was successfully employed for the description of the inhibitory interaction among the degradation process of the mixture of tetrahydrofuran, ethylbenzene, benzene, and toluene by $P$. oleovorans DT4 (Chen et al. 2014). Carvajal et al. found that the modified Gompertz model could be well employed to study the antagonistic interactions among benzene, toluene, ethylbenzene, and xylene, when single and dual substrates were degraded by the activated sludge under anoxic condition (Carvajal et al. 2018).

In this study, the biodegradation performance of malodorous pollutants PT and DMS by the bacterial strains P. putida S-1 and Alcaligenes sp. SY1 was investigated. The interactions between PT and DMS in a dual-substrate biodegradation system were explored, the cause of long lag in DMS degradation was carefully analyzed, and a modified Gompertz model was developed to characterize the simultaneous degradation behavior of PT and DMS. It is believed that the mechanism of the lag phase proposed here may inspire more rational operation to obtain superior simultaneous removal efficiency of multiple gaseous pollutants coexisted in practical situations.

\section{Materials And Methods}

\subsection{Chemicals and reagents}

The chemicals used for culture were of analytical grade, the chemicals/solvents used for gas chromatographic analysis were of chromatographic grade, and the odorant compounds DMS and PT were in liquid form (>99\%). The mineral medium (MM) used in the batch experiment was composed of $4.5 \mathrm{~g} \cdot \mathrm{L}^{-1} \mathrm{Na}_{2} \mathrm{HPO}_{4} \cdot 12 \mathrm{H}_{2} \mathrm{O}, 1.0 \mathrm{~g} \cdot \mathrm{L}^{-1} \mathrm{KH}_{2} \mathrm{PO}_{4}, 1.5 \mathrm{~g} \cdot \mathrm{L}^{-1} \mathrm{NH}_{4} \mathrm{Cl}, 0.023 \mathrm{~g} \cdot \mathrm{L}^{-1} \mathrm{CaCl}_{2}, 0.2 \mathrm{~g} \cdot \mathrm{L}^{-1} \mathrm{MgCl}_{2}$, and 1 $\mathrm{mL}$ of trace element stock solution. The trace element stock solution contained $1.0 \mathrm{~g} \cdot \mathrm{L}^{-1} \mathrm{FeSO}_{4} \cdot 7 \mathrm{H}_{2} \mathrm{O}$, $0.02 \mathrm{~g} \cdot \mathrm{L}^{-1} \mathrm{CuSO}_{4} \cdot 5 \mathrm{H}_{2} \mathrm{O}, 0.014 \mathrm{~g} \cdot \mathrm{L}^{-1} \mathrm{H}_{3} \mathrm{BO}_{3}, 0.10 \mathrm{~g} \cdot \mathrm{L}^{-1} \mathrm{MnSO}_{4} \cdot 4 \mathrm{H}_{2} \mathrm{O}, 0.10 \mathrm{~g} \cdot \mathrm{L}^{-1} \mathrm{ZnSO}_{4} \cdot 7 \mathrm{H}_{2} \mathrm{O}, 0.02 \mathrm{~g} \cdot \mathrm{L}^{-1}$ 
$\mathrm{Na}_{2} \mathrm{MnO}_{4} \cdot 2 \mathrm{H}_{2} \mathrm{O}$, and $0.02 \mathrm{~g} \cdot \mathrm{L}^{-1} \mathrm{CoCl}_{2} \cdot 6 \mathrm{H}_{2} \mathrm{O}$. The $\mathrm{pH}$ of the medium was adjusted to 7.0 by using phosphate buffer solution. All chemicals and reagents were purchased from Sigma-Aldrich.

\subsection{Microorganisms and culture conditions}

Alcaligenes sp. SY1, a Gram-negative aerobic heterotrophic bacterium with DMS-degrading ability, was isolated from activated sludge collected from a pharmaceutical factory (Taizhou, China) (Sun et al. 2016). P. putida S-1 was reported as the first $P$. putida strain capable of degrading malodorous PT (Chen et al. 2016). These two strains were stored in China Center for Type Culture Collection under CTCC Nos. M 2014619 and M 2013444, and their 16S rRNA sequences were deposited in the GenBank database under Accession Nos. KP162176 and KF640247, respectively. For inoculum preparation, P. putida S-1 was initially incubated in R2A liquid medium, and Alcaligenes sp. SY1 was incubated in MM with $50 \mathrm{mg} \cdot \mathrm{L}^{-1}$ DMS. Both strains were incubated on a rotary shaker $(160 \mathrm{rpm})$ at $30^{\circ} \mathrm{C}$.

\subsection{Batch biodegradation experiment}

All batch biodegradation experiments were carried out in $250 \mathrm{~mL}$ screw-capped Erlenmeyer flasks containing $50 \mathrm{~mL}$ of sterile MM with different initial PT and/or DMS concentrations. The bacterial inoculums were obtained at exponential growth phase by centrifugation at $6000 \mathrm{rpm}$ for $10 \mathrm{~min}$ at $4^{\circ} \mathrm{C}$, washed with MM twice, and resuspended in MM at an optical density (OD600) of 0.1. To ensure the same initial biomass concentration of both strains, we introduced $0.94 \mathrm{~mL}$ of $P$. putida S-1 suspension and 1 $\mathrm{mL}$ of Alcaligenes sp. SY1 suspension. When presented as the sole carbon source in the medium, $>300$ $\mathrm{mg} \cdot \mathrm{L}^{-1}$ of PT inhibited the growth of $P$. putida S-1, and high initial DMS $\left(>200 \mathrm{mg} \cdot \mathrm{L}^{-1}\right)$ concentration inhibited the growth of Alcaligenes sp. SY1. The concentrations of PT and/or DMS below the minimum inhibitory concentration were selected in our batch biodegradation experiments. The initial substrate concentrations were $50 \mathrm{mg} \cdot \mathrm{L}^{-1}$ unless otherwise specified, and the introduced volumes of pregrown cells were consistent with the above-mentioned conditions.

During the experiments, the concentrations of substrates and biomass were determined at regular time intervals until complete degradation. The initial concentrations of the substrates remained unchanged in the control experiments without the strains, implying that abiotic loss could be neglected. All experiments were performed in triplicate.

\subsection{Analytical methods}

The residual concentrations of PT and DMS were determined by gas chromatography (SHIMADZU GC14B, JAPAN) equipped with a flame ionization detector and an RTX-1 column (30 m $\times 0.32 \mathrm{~mm} \times 25 \mu \mathrm{m}$, Restek, USA). PT and DMS could be balanced in vapor and aqueous phase in the sealed bottle within 30 $\min$. Then, the aqueous concentrations of substrates were calculated from the measured concentration in the gas phase by using the partition coefficient (Henry's coefficient). The gas samples $(0.8 \mathrm{~mL})$ in the headspace were withdrawn with a gas-tight syringe and injected into the GC to determine the gaseous concentrations of PT and DMS. The temperatures of GC injector, oven, and detector were $200^{\circ} \mathrm{C}, 80^{\circ} \mathrm{C}$, and $230^{\circ} \mathrm{C}$, respectively. The hydrogen and air flow rates for FID were 40 and $450 \mathrm{~mL} \cdot \mathrm{min}^{-1}$, respectively. 
The $\mathrm{CO}_{2}$ concentration was determined by gas chromatography (Agilent Technologies, GC $6890 \mathrm{~N}$, USA) equipped with HP-Plot-Q column $(30 \mathrm{~m} \times 0.32 \mathrm{~mm} \times 20 \mu \mathrm{m})$ and thermal conductivity detector. The carrier gas was helium with a flow rate of $5 \mathrm{~mL} \mathrm{~min}{ }^{-1}$. The column and detector temperatures were $40^{\circ} \mathrm{C}$ and $100^{\circ} \mathrm{C}$, respectively.

The concentration of $\mathrm{SO}_{4}{ }^{2-}$ was determined with an ICS 2000 ion chromatograph equipped with lonpac AS19-HC separation column and DS conductance detector. The flow rate of $\mathrm{KOH}$ was $1.0 \mathrm{~mL} \cdot \mathrm{min}^{-1}$, and the column temperature was $30^{\circ} \mathrm{C}$.

The intermediates of PT degradation were identified using a gas chromatograph-mass spectrometer (GC-MS, Agilent 7890N/MS 5975) equipped with an HP-5MS capillary column, as described elsewhere (Chen et al. 2016). Solid-phase extraction with a C18 column was adopted for sample preparation.

Biomass concentrations were monitored by spectrophotometric absorbance at $600 \mathrm{~nm}\left(\mathrm{OD}_{600}\right)$ on a spectrophotometer (Hitachi, Tokyo).

\subsection{Kinetic modeling}

In this study, the description of the degradation kinetics of the substrates (PT and DMS) under different conditions is the main content of modeling. Among the mathematical models used to describe biodegradation kinetics, the pseudo first-order model was applied in many cases (Elango, Kurtz, and Freedman 2011; Boonnorat et al. 2014). It is defined as follows:

$$
S_{t}=S_{O} e^{-q t}
$$

where $q$ is the degradation rate constant of the substrate $\left(\mathrm{h}^{-1}\right), S_{O}$ is the initial aqueous concentration of the substrate, $S_{t}$ is the substrate concentration in the liquid at time $(t)$, and $t$ is the reaction time.

However, substrate interactions caused by the presence of co-substrate increase the uncertainty in the degradation process. Thus, some of the modified versions of general models that may provide an improved fit to biodegradation must be considered. In food microbiology, several nonlinear regression functions, such as the Gompertz model, have been widely used in describing microbial growth or production. Zwietering et al. reparameterized the Gompertz function through replacing nonsensical mathematical parameters with those with biological meaning and developed the Gompertz Kinetic model, providing a function of the cumulative production $(P)$ with time (Equation 2) (Zwietering et al. 1990).

$$
\mathrm{P}(\mathrm{t})=P_{\max } \exp \left(-\exp \left(\frac{R_{m}}{P_{\max }}(\lambda-\mathrm{t})+1\right)\right)
$$

where $P_{\max }$ is the maximum production, $R_{m}$ is the maximum rate of production, $\lambda$ is the lag phase, and $t$ is the elapsed time. The modified Gompertz kinetic model with the incorporation of parameter $\lambda$ 
provides the probability of modeling biodegradation process with lag.

The new variable cumulative degradation $D$ and maximum degradation potential $D_{\text {max }}$ were used in describing substrate consumption as a degradation potential (Equation 3) (Carvajal et al. 2018). In this present study, the experimental data in the degradation systems with two substrates may be fitted to the modified Gompertz kinetic model.

$$
\begin{gathered}
D_{(t)}=D_{\max } \exp \left(-\exp \left(\frac{R_{m}}{D_{\max }}(\lambda-t)+1\right)\right) \\
D_{(t)}=\frac{C_{0}-C_{t}}{c_{0}}
\end{gathered}
$$

Experimental data were fitted to the models with a nonlinear curve fitting to estimate the kinetic parameters of the models used. For the pseudo first-order model, substrate concentration versus time was directly fitted, and the degradation rate constant $q$ could be obtained. In the modified Gompertz model, the cumulative degradation at $t\left(D_{(t)}\right)$ was calculated using Equation (4), where $C_{0}$ and $C_{t}$ indicate the initial substrate concentration and substrate concentration as a function of time, respectively.

1stOpt statistical analysis software was used for parameter determination (Zhang et al. 2013). Levenberg-Marquardt and universal global optimization algorithms were used for calculating the correlation coefficient $\left(R^{2}\right)$.

\section{Results And Discussion}

\subsection{Long lag occurred in DMS degradation in binary mixture}

Figure 1 showed the individual and simultaneous biodegradation performance of $50 \mathrm{mg} \cdot \mathrm{L}^{-1} \mathrm{PT}$ and 50 $\mathrm{mg} \cdot \mathrm{L}^{-1}$ DMS by the bacterial strains of $P$. putida S-1 and Alcaligenes sp. SY1. And the cell growth was also recorded. It could be seen that PT was degraded completely in $33 \mathrm{~h}$ almost without lag phase, regardless of the presence of DMS. This indicated that the degradation of PT was not affected by the presence of DMS. By contrast, it was not until $81 \mathrm{~h}$ later that DMS began to be biodegraded in the presence of PT. And only a lag of approximately $10 \mathrm{~h}$ was observed when Pt was absent (Fig. 1a). Similar phenomenon in double-substrate system were also previously reported (Chen et al. 2007; Hsieh et al. 2008). The biomass concentration expressed in $\mathrm{OD}_{600}$ increased with the degradation of $P T$, and then the growth ceased and remained for a long time until the degradation of DMS started. Similar diauxic-growth phenomena had also been observed in previous studies, when bacteria switched between electron donors (Monod 1949; Lisbon et al. 2002). Notably, biomass growth continued, even after PT was completely degraded, while the degradation of DMS was far from starting. Besides, the concentrations of $\mathrm{CO}_{2}$ and $\mathrm{SO}_{4}{ }^{2-}$ kept on increasing, even though PT was completely depleted (Fig. 1b), which was probably ascribed to the further mineralization of the intermediates of PT. 
Subsequently, the influence of the substance concentration on the degradation performance of PT and DMS was investigated. As shown in Table 1, when the concentration of DMS was fixed at $50 \mathrm{mg} \cdot \mathrm{L}^{-1}$, and the concentration of PT was varied at $0,10,20,50$, and $100 \mathrm{mg} \cdot \mathrm{L}^{-1}$, a lag of $11-117 \mathrm{~h}$ was observed before the onset of DMS degradation, and the lag duration was extended with the increase of PT concentration. When the concentration of PT in the dual-substrate mixture was $100 \mathrm{mg} \cdot \mathrm{L}^{-1}$, its degradation efficiency could reach $90 \%$ in $28 \mathrm{~h}$, however, the lag phase for DMS degradation was long as $117 \mathrm{~h}$. The actual degradation times required for the $90 \%$ DMS removal ranged from $24-48 \mathrm{~h}$. When the PT concentration was $10 \mathrm{mg} \cdot \mathrm{L}^{-1}, 90 \%$ DMS could be degraded in $24 \mathrm{~h}$, which was even shorter than that without PT (34 h). By contrast, when the initial PT concentration was increased to $50 \mathrm{mg}^{-\mathrm{L}^{-1}}$, the actual degradation time for $90 \%$ DMS removal prolonged to $48 \mathrm{~h}$. Thus, it could be concluded that PT concentration influence the enhancement or the inhibitory effect on DMS degradation, when the lag was not considered.

Table 1

Biodegradation of different initial concentrations of PT and $50 \mathrm{mg} \cdot \mathrm{L}^{-1}$ DMS by $P$. putida S-1 and Alcaligenes sp. SY1 in liquid culture.

\begin{tabular}{|c|c|c|c|c|c|c|}
\hline $\begin{array}{l}\text { Initial } \\
\text { PT } \\
\text { (mg/L) }\end{array}$ & $\begin{array}{l}\text { Initial } \\
\text { DMS } \\
\text { (mg/L) }\end{array}$ & $\begin{array}{l}\text { Time for } \\
90 \% \mathrm{PT} \\
\text { removal (h) }\end{array}$ & $\begin{array}{l}\text { Lag to DMS } \\
\text { degradation } \\
\text { (h) }\end{array}$ & $\begin{array}{l}\text { Time for } 90 \% \\
\text { DMS removal } \\
\text { (h) }\end{array}$ & $\begin{array}{l}\text { OD }_{600} \text { when } \\
90 \% \text { PT } \\
\text { removed }\end{array}$ & $\begin{array}{l}\mathrm{OD}_{600} \text { when } \\
90 \% \text { DMS } \\
\text { removed }\end{array}$ \\
\hline 0 & 50 & I & 11 & 45 & I & 0.075 \\
\hline 10 & 50 & 8 & 33 & 57 & 0.018 & 0.080 \\
\hline 20 & 50 & 11 & 57 & 93 & 0.037 & 0.96 \\
\hline 50 & 50 & 21 & 81 & 129 & 0.051 & 0.108 \\
\hline 100 & 50 & 28 & 117 & 153 & 0.085 & 0.148 \\
\hline
\end{tabular}

\subsection{The cause of the long DMS-degrading lag}

To find out the cause of such lag-related inhibition, several control experiments were conducted.

Firstly, the degradation performance of DMS in presence of sole Alcaligenes sp. SY1 and both strains were investigated. As displayed in Fig. 2, the degradation efficiencies in both cases were analogous that DMS was biodegraded completely in $60 \mathrm{~h}$, which indicated that the presence of $P$. putida S-1 strain was not the cause of the lag phase during DMS degradation.

Besides, it has been widely reported that the formation and accumulation of intermediates of one substrate can cast inhibited influence on the degradation of another one (Hazrati, Shayegan, and Seyedi 2015; Reardon, Mosteller, and Rogers 2015). Since DMS could be degraded after a long lag duration, when PT and its degrader P. putida S-1 were co-present in the medium. This suggested that Alcaligenes sp. SY1 could survive in the possible soluble antagonistic compounds secreted by $P$. putida S-1, or the concentration of antagonistic compounds might be low to completely kill Alcaligenes sp. SY1. Then the 
components and concentrations of the intermediates were detected, when PT was completely degraded by P. putida S-1 (at $32 \mathrm{~h}$ of the whole process). The results showed that PT could be degraded into dipropyl disulfide, 3-hexanol, 2-hexanol, 3-hexanone, and 2-hexanone, and their accumulated concentrations in the solutions were all lower than $5 \mathrm{mg} \cdot \mathrm{L}^{-1}$ (see Table 2). And as the degradation time extended, such intermediates could be further degraded, resulting in the concentrations being less than $0.1 \mathrm{mg} \cdot \mathrm{L}^{-1}$ after $58 \mathrm{~h}$ of degradation. Moreover, the variation trend of DMS degradation was almost identical with the results of control group except for the lag, when PT was completely depleted, intermediates were remained, and the biomass of $P$. putida S-1 was withdrawn from the liquid.

Furthermore, it was found that the addition of every intermediates of PT with a concentration of $5 \mathrm{mg} \cdot \mathrm{L}^{-1}$ did not increase the lag phase of DMS degradation (data not shown). All these results robustly proved that Alcaligenes sp. SY1 could only live by DMS, and the metabolites of PT degradation by P. putida S-1 were unrelated to the prolonged DMS-degrading lag.

Delightedly, when PT was present in the medium and P. putida S-1 was not added, DMS could not be degraded by Alcaligenes sp. SY1. This suggested that PT itself may cast a detrimental effect on the degradation of DMS. To confirm this speculation, Alcaligenes sp. SY1 strains were exposed to $50 \mathrm{mg} \cdot \mathrm{L}^{-1}$ PT for 24,48 , and $72 \mathrm{~h}$. Then the cells were collected by centrifugation, washed and resuspended for DMS degradation in fresh MM. As shown in Fig. 3, lag lasted approximately 90, 110, and $140 \mathrm{~h}$, respectively, and the longer the times exposed to PT, the longer the lag phase would last. And the lag durations were even longer than that observed in Fig. 1b, which was consistent with the results in Table 1. Therefore, it could be concluded that the concentration of PT and the exposure time of Alcaligenes sp. SY1 to PT are concerned with the lag duration of DMS metabolism in the mixture.

Table 2

Variation of the intermediates that accumulated in the solution after PT depletion under the dualsubstrate condition.

\begin{tabular}{|llllll|}
\hline $\begin{array}{l}\text { Time } \\
(\mathrm{h})\end{array}$ & $\begin{array}{l}\text { Dipropyl disulfide } \\
(\mathrm{mg} / \mathrm{L})\end{array}$ & $\begin{array}{l}\text { 3-Hexanol } \\
(\mathrm{mg} / \mathrm{L})\end{array}$ & $\begin{array}{l}\text { 2-Hexanol } \\
(\mathrm{mg} / \mathrm{L})\end{array}$ & $\begin{array}{l}\text { 3-Hexanone } \\
(\mathrm{mg} / \mathrm{L})\end{array}$ & $\begin{array}{l}\text { 2-Hexanone } \\
(\mathrm{mg} / \mathrm{L})\end{array}$ \\
\hline 32 & $4.23 \pm 1.10$ & $0.65 \pm 0.14$ & $0.57 \pm 0.19$ & $1.13 \pm 0.26$ & $0.92 \pm 0.22$ \\
\hline 45 & $2.11 \pm 1.25$ & $<0.1$ & $<0.1$ & $0.21 \pm 0.11$ & $0.25 \pm 0.13$ \\
\hline 58 & $<0.1$ & $<0.1$ & $<0.1$ & $<0.1$ & $<0.1$ \\
\hline
\end{tabular}

\subsection{Citric acid acted as an effective "lag reductor"}

It is of great importance to shorten the lag phase of DMS degradation in a dual-substrate solution (Chen et al. 2018). Adding extra carbon sources can probably enhance the degradation rate of pollutants (Mukherjee and Bordoloi 2012). Some readily metabolized organic substrates, such as yeast extract, tryptone, and glucose, might stimulate biomass growth, thereby improving the mineralization of recalcitrant organic compounds (Chen, Chen, and Zhong 2009; Ziagova and Liakopoulou-Kyriakides 2007). It was also reported that benzene degradation could greatly enhanced by tetrahydrofuran, which 
could act as an "energy generator" (Zhou et al. 2011). Hence, to reduce the lag phase for DMS degradation, various organic substrates, like yeast extract, tryptone, methanol, starch, acetone, and sucrose, were added and their influence on DMS degradation was investigated and compared. As shown in Fig. 4a, glucose, yeast extract, and citric acid were all beneficial for the removal of DMS when PT coexisted. And the addition of these three carbon sources exhibited varying reduction for the lag phase. Citric acid demonstrated the optimal performance in decreasing the lag phase, only $24 \mathrm{~h}$ of lag was observed before the onset of DMS degradation. Nevertheless, the DMS biodegradation rates after lag phase under different conditions were similar with each other, regardless of the presence and kinds of the supplements. However, the cause behind the superior effect of citric acid on lag reduction was complex and remains unknown.

The addition of extra carbon sources could also enhance the biodegradation of PT, as shown in Fig. 4b, which may shorten the exposure time of Alcaligenes sp. SY1 to PT and thus reduce the lag phase for DMS degradation. However, the stimulation effect toward PT degradation upon the addition of citric acid was not better than those upon glucose and yeast extract. As an easily metabolized organic substrate, citric acid could also provide additional energy for DMS degradation, analogous to that of glucose and yeast extract. Besides, it is the main substrate in Kreb's cycle and hence probably producing energy more easily. Moreover, Mahmud et al. found that citric acid could enhance the tolerance of Brassica juncea to cadmium toxicity through upregulating the antioxidant defense and glyoxalase systems (Al Mahmud et al. 2018), thus we speculate that citric acid may also improve the tolerance of Alcaligenes sp. SY1 to PT toxicity.

\subsection{Kinetics of PT and DMS biodegradation under single and binary substrate conditions}

Pseudo first-order model was first used to describe the biodegradation of substrates. The fitness of the first-order model for PT degradation in the dual-substrate biodegradation tests was analogous to that obtained under the single-substrate condition (Tables 3 and 4). The $R^{2}$ values obtained for the degradation of PT at low concentrations (0.931 and 0.926$)$ were relatively higher than that obtained at high concentrations $(0.892$ and 0.895$)$. Under the dual-substrate condition, when the PT concentration exceeded $10 \mathrm{mg} \cdot \mathrm{L}^{-1}$, the model provided poor fitting results $\left(R^{2}<0.9\right)$. As shown in Fig. 5 , the fitting curves matched well with the experimental results in the first few hours, but it was then gradually deviated as the biodegradation proceeded, when PT biodegradation was fitted with logarithmic mode. When the initial PT concentrations were $10,20,50$, and $100 \mathrm{mg} \cdot \mathrm{L}^{-1}$, the values of the degradation rate constant $q$ obtained for PT degradation under the single-substrate condition were $0.16,0.09,0.04$, and $0.03 \mathrm{~h}^{-1}$, respectively. A negative correlation was found between $q$ and PT concentration. The $q$ obtained at every PT concentration under the dual-substrate condition were slightly higher, which indicated that the presence of DMS could slightly enhance the degradation of PT. 
Unfortunately, the pseudo first-order model failed to predict the kinetics of DMS biodegradation under the dual-substrate condition. When PT and its degrader P. putida S-1 were co-present in the medium, the lag phase accounted for more than $50 \%$ of the whole time of DMS degradation before the onset of DMS degradation. Such lag phase made the modeling of the DMS removal process difficult to describe. Therefore, the lag phase must be excluded, and then, the Monod model was adopted for the description of microbial growth (Strigul, Dette, and Melas 2009). Meanwhile, as the lag phase mattered the completion of pollutant elimination, it also needed to be predicted. Nevertheless, conventional mathematical models, such as the first-order model, Andrews model and SKIP model, which account for the inhibition between dual substrate, failed to fit the experimental data in this study (data not shown). Encouragingly, Christen et al. used the Gompertz model to calculate the maximum degradation rate of phenol, and the results were well fitted $\left(R^{2}>0.98\right)$ (Christen et al. 2012). Inspired by this, the Gompertz model was then adopted and modified by incorporating $\lambda$ as the lag phase, to describe the biodegradation of DMS (Table 5 and Fig. 6 ). The $R^{2}$ was higher than 0.97 , and the maximum degradation potential $\left(D_{\max }\right)$ reached 1.00, confirming the thorough degradation of PT and DMS in the whole degradation process. The obtained lag phase parameters $(\lambda)$ of DMS degradation were $16.19,35.59$, 69.38, 90.46, and 117.08 h, when mixed with PT at initial concentrations of $0,10,20,50$, and $100 \mathrm{mg} \cdot \mathrm{L}^{-1}$, respectively. All these values were in good accordance with the experimental lag phase of DMS degradation illustrated in Table 1.

Table 3

Kinetics parameters estimated by the modified Gompertz model and the first-order kinetic model for the degradation of PT with different initial concentrations under single-substrate condition.

\begin{tabular}{|lllllll|}
\hline $\begin{array}{l}\text { PT concentration } \\
(\mathrm{mg} / \mathrm{L})\end{array}$ & \multicolumn{2}{l}{ The Modified Gompertz model } & \multicolumn{3}{l|}{ The first-order kinetic model } \\
\cline { 2 - 7 } & $\boldsymbol{D}_{\text {max }}(\mathrm{mg} / \mathrm{mg})$ & $\boldsymbol{R}_{\boldsymbol{m}}$ & $\boldsymbol{\lambda}$ & $\mathrm{R}^{2}$ & $\boldsymbol{q}$ & $\mathrm{R}^{2}$ \\
$\mathbf{( m g / ( m g \cdot h ) )}$ & $(\mathrm{h})$ & & $(1 / \mathrm{h})$ & \\
\hline 10 & 1.00 & 0.48 & 2.32 & 0.996 & 0.16 & 0.931 \\
\hline 50 & 1.00 & 0.32 & 4.20 & 0.998 & 0.09 & 0.926 \\
\hline 100 & 1.00 & 0.15 & 5.88 & 0.998 & 0.04 & 0.892 \\
\hline
\end{tabular}

The $\lambda$ values at 2.32, 4.20, 5.88, and $6.44 \mathrm{~h}$ were recorded for PT degradation at concentrations of 10, 20, 50 , and $100 \mathrm{mg} \cdot \mathrm{L}^{-1}$ (Table 3 ) in single-substrate solution. The correlations between $\lambda$ and PT concentration were consistent with that reported in previous studies, i.e., the lag phase increased with the initial substrate concentration (Juang and Tsai 2006; Christen et al. 2012). Notably, the barely detectable lag phase of PT degradation could also be calculated using the modified Gompertz model.

Compared with those in single-substrate solution, the $\lambda$ values of PT degradation were relatively lower and the maximum degradation rates $R_{m}$ were relatively higher when the reaction was carried out in dual- 
substrate conditions, regardless of its initial concentration (Table 4). This indicated that PT degradation could be promoted in the presence of DMS, similar to that obtained using the first-order model in terms of the degradation rate constant $q$.

Table 4

Kinetics parameters estimated with the modified Gompertz model and the first-order kinetic model for the degradation of different initial concentrations of PT under dual-substrate conditions with $50 \mathrm{mg} \cdot \mathrm{L}^{-1} \mathrm{DMS}$.

\begin{tabular}{|c|c|c|c|c|c|c|}
\hline \multirow{2}{*}{$\begin{array}{l}\text { PT concentration } \\
(\mathrm{mg} / \mathrm{L})\end{array}$} & \multicolumn{4}{|c|}{ The Modified Gompertz model } & \multicolumn{2}{|c|}{ The first-order kinetic model } \\
\hline & $\begin{array}{l}D_{\max } \\
(\mathrm{mg} / \mathrm{mg})\end{array}$ & $\begin{array}{l}R_{m} \\
(\mathrm{mg} /(\mathrm{mg} \cdot \mathrm{h}))\end{array}$ & $\begin{array}{l}\lambda \\
\text { (h) }\end{array}$ & $\mathbf{R}^{2}$ & $\begin{array}{l}q \\
(1 / h)\end{array}$ & $\mathbf{R}^{2}$ \\
\hline 10 & 1.00 & 0.51 & 1.86 & 0.993 & 0.18 & 0.936 \\
\hline 20 & 1.00 & 0.38 & 3.94 & 0.982 & 0.10 & 0.884 \\
\hline 50 & 1.00 & 0.16 & 5.21 & 0.979 & 0.05 & 0.873 \\
\hline 100 & 1.00 & 0.12 & 5.68 & 0.970 & 0.04 & 0.895 \\
\hline
\end{tabular}

For DMS biodegradation, the $R_{m}$ values were calculated to be $0.17,0.13,0.07$, and $0.09 \mathrm{mg} \cdot \mathrm{mg}^{-1} \cdot \mathrm{h}^{-1}$ in the presence of $10,20,50$, and $100 \mathrm{mg} \cdot \mathrm{L}^{-1} \mathrm{PT}$, respectively, but was $0.13 \mathrm{mg} \cdot \mathrm{mg}^{-1} \cdot \mathrm{h}^{-1}$ without PT addition (Table 5). When the concentration of the co-substrate PT was $10 \mathrm{mg}^{\cdot \mathrm{L}^{-1}}$, the $R_{m}$ of DMS biodegradation was higher than that without PT. It could thus be inferred that, if PT-trigged lag was not considered, a slight enhancement for DMS biodegradation after PT addition could be achieved at a low PT concentration (e.g. $10 \mathrm{mg} \cdot \mathrm{L}^{-1}$ ). Such enhancement might be attributed to the metabolism of the degradation intermediate of DMS by PT-grown cells of $P$. putida S-1. This was the first study reported that only lag prolongation, rather than degradation rate reduction, accounted for the biodegradation inhibition of one pollutant by the other in a binary mixture. However, A PT concentration higher than $20 \mathrm{mg} \cdot \mathrm{L}^{-1}$ could lead to lower $R_{m}$ of DMS biodegradation. Two probable reasons may account for such results. One was the competition between Alcaligenes sp. SY1 and P. putida S-1 for the limited mineral elements during PT degradation; the other was the inhibition of high-concentration PT to the enzyme activity responsible for DMS biodegradation. 
Table 5

Kinetics parameters estimated with the modified Gompertz model for the degradation of $50 \mathrm{mg} \cdot \mathrm{L}-1$ DMS in the dual-substrate solution with different initial concentrations of PT.

\begin{tabular}{|lllll|}
\hline PT concentration $(\mathrm{mg} / \mathrm{L})$ & $\begin{array}{l}\boldsymbol{D}_{\max } \\
(\mathrm{mg} / \mathrm{mg})\end{array}$ & $\begin{array}{l}\boldsymbol{R}_{\boldsymbol{m}} \\
(\mathrm{mg} /(\mathrm{mg} \cdot \mathrm{h}))\end{array}$ & $\begin{array}{l}\lambda \\
(\mathrm{h})\end{array}$ & $\mathrm{R}^{2}$ \\
\hline 0 & 1.00 & 0.13 & 16.19 & 0.988 \\
\hline 10 & 1.00 & 0.17 & 36.59 & 0.996 \\
\hline 20 & 1.00 & 0.13 & 69.38 & 0.990 \\
\hline 100 & 1.00 & 0.07 & 90.46 & 0.992 \\
\hline
\end{tabular}

\section{Conclusion}

In this study, simultaneous biodegradation of malodorous 1-propanethiol (PT) and dimethyl sulfide (DMS) with Pseudomonas putida S-1 and Alcaligenes sp. SY1 were investigated and related interactions were explored. PT was completely degraded in $33 \mathrm{~h}$, while a lag of $10 \mathrm{~h}$ was observed for DMS degradation, and the lag was even extended to $81 \mathrm{~h}$ in the mixture. Careful analysis found that the lag was mainly attributed to the exposure of DMS degrader (Alcaligenes sp. SY1) to PT, rather than the PT metabolites and PT degrader. And the exposure time and PT concentration largely influenced the lag duration. Citric acid could serve as an effective "lag reductor", decreasing the lag to $24 \mathrm{~h}$. Pseudo firstorder model was proved suitable for the description of PT degradation, revealing that the degradation of PT could be enhanced in the presence of DMS regardless of its concentration. A modified Gompertz model incorporated the lag phase parameter $\lambda$ was developed for the successful description of DMS degradation in the presence of PT. And the effect of PT on DMS degradation depended on its initial concentration. When the PT-triggered lag was not considered, low-concentration PT could promote the biodegradation of DMS, while a higher concentration ( $\left.\nabla 20 \mathrm{mg} \cdot \mathrm{L}^{-1}\right)$ could cast negative effect. This is the first report that only lag prolongation, rather than degradation rate reduction, accounted for the biodegradation inhibition of one pollutant to the other. It is believed that the mechanism proposed in this work may inspire more rational operation to obtain superior simultaneous removal efficiency of multiple gaseous pollutants coexisted in practical situations.

\section{Declarations}

\section{Ethics approval and consent to participate}

Not applicable.

\section{Consent for publication}


Not applicable.

\section{Availability of data and materials}

The datasets used and/or analyzed during the current study are available from the corresponding author on reasonable request.

\section{Competing interests}

The authors declare that they have no known competing financial interests or personal relationships that could have appeared to influence the work reported in this paper.

\section{Funding}

This work was financially supported by the National Natural Science Foundation of China (NSFC51778581, NSFC-52070169).

\section{Author Contributions}

QL was a major contributor in investigation, paper writing and revision; ZT performed the experiments and data analysis; JZ participated in the completion of the experiments; JH participated in the data analysis; JC contributed to the resources and funding; DC made an important contribution to the resources, funding and supervision. All authors have read and approved the final manuscript.

\section{References}

1. Al Mahmud J, Hasanuzzaman M, Nahar K, Bhuyan MHMB, Fujita M (2018) Insights into citric acidinduced cadmium tolerance and phytoremediation in Brassica juncea L.: Coordinated functions of metal chelation, antioxidant defense and glyoxalase systems. Ecotoxicol Environ Saf 147:990-1001

2. Bielefeldt AR, Cort T (2005) Dual substrate biodegradation of a nonionic surfactant and pentachlorophenol by Sphingomonas chlorophenolica RA2. Biotechnol Bioeng 89:680-9

3. Boonnorat J, Chiemchaisri C, Chiemchaisri W, Yamamoto K (2014) Removals of phenolic compounds and phthalic acid esters in landfill leachate by microbial sludge of two-stage membrane bioreactor. J Hazard Mater 277:93-101

4. Carvajal A, Akmirza I, Navia D, Pérez R, Muñoz R, Lebrero R (2018) Anoxic denitrification of BTEX: Biodegradation kinetics and pollutant interactions. J Environ Manage 214:125-136

5. Chen DZ, Chen JM, Zhong WH (2009) Enhancement of methyl tert-butyl ether degradation by the addition of readily metabolizable organic substrates. J Hazard Mater 167:860-865

6. Chen D, Zhi YF, Ding YY, Zhou JX, Ye, and Jian Meng Chen (2014) Biodegradation kinetics of tetrahydrofuran, benzene, toluene, and ethylbenzene as multi-substrate by Pseudomonas oleovorans DT4. Int J Environ Res Public Health 12:371-384 
7. Chen D, Zhi YM, Han SLiM, Chen J, Ye JX, Jian Meng Chen (2016) A newly isolated Pseudomonas putida S-1 strain for batch-mode-propanethiol degradation and continuous treatment of propanethiol-containing waste gas. J Hazard Mater 302:232-240

8. Chen D, Zhi XY, Zhao XP, Miao J, Chen JX, Ye ZW, Chen SH, Zhang, and Jian Meng Chen (2018) A solid composite microbial inoculant for the simultaneous removal of volatile organic sulfide compounds: Preparation, characterization, and its bioaugmentation of a biotrickling filter. J Hazard Mater 342:589-596

9. Chen Y, Min TF, Lin C, Huang, Che J Lin (2008) Cometabolic degradation kinetics of TCE and phenol by Pseudomonas putida. Chemosphere 72:1671-1680

10. Chen Y, Min TF, Lin C, Huang JC, Lin, Feng Ming Hsieh (2007) Degradation of phenol and TCE using suspended and chitosan-bead immobilized Pseudomonas putida. J Hazard Mater 148:660-670

11. Chignell JF, Park S, Lacerda CMR, De Long SK, Reardon KF (2018) Label-Free Proteomics of a Defined, Binary Co-culture Reveals Diversity of Competitive Responses Between Members of a Model Soil Microbial System. Microb Ecol 75:701-719

12. Christen P, Vega A, Casalot L, Simon G, Auria R (2012) Kinetics of aerobic phenol biodegradation by the acidophilic and hyperthermophilic archaeon Sulfolobus solfataricus 98/2. Biochem Eng $J$ 62:56-61

13. Durme J, Van J, Dewulf C, Leys (2008) Combining non-thermal plasma with heterogeneous catalysis in waste gas treatment: A review. Appl Catal B 78:324-333Van Langenhove (

14. Elango V, Kurtz HD, Freedman DL (2011) Aerobic cometabolism of trichloroethene and cisdichloroethene with benzene and chlorinated benzenes as growth substrates. Chemosphere 84:247253

15. Giri B, Shekher KH, Kim RA, Pandey J, Cho H, Song, Yoon Shin Kim (2014) Review of biotreatment techniques for volatile sulfur compounds with an emphasis on dimethyl sulfide. Process Biochem 49:1543-1554

16. Giri B, Shekher, Pandey RA (2013) Biological treatment of gaseous emissions containing dimethyl sulphide generated from pulp and paper industry. Bioresour Technol 142:420-427

17. Han B, Liu YT, Wu JH, Yin Chang F (2018) Characterization of industrial odor sources in Binhai New Area of Tianjin, China. Environ Sci Pollut Res Int 25:14006-14017

18. Hazrati H, Shayegan J, Seyed Mojtaba Seyedi (2015) Biodegradation kinetics and interactions of styrene and ethylbenzene as single and dual substrates for a mixed bacterial culture. J Environ Health Sci Eng 13:1-12

19. Hsieh F, Ming C, Huang TF, Lin YM, Chen, Jui Che Lin (2008) Study of sodium tripolyphosphatecrosslinked chitosan beads entrapped with Pseudomonas putida for phenol degradation. Process Biochem 43:83-92

20. Jo WK, Myeong H, Shin (2010) Applicability of a continuous-flow system inner-coated with S-doped titania for the photocatalysis of dimethyl sulfide at low concentrations. J Environ Manage 91:20592065 
21. Juang R, Shin, Shang Yuan Tsai (2006) Growth kinetics of Pseudomonas putida in the biodegradation of single and mixed phenol and sodium salicylate. Biochem Eng J 31:133-140

22. Kennes C, Veiga MC (2013) Air Pollution Prevention and Control: Bioreactors and Bioenergy. John Wiley \& Sons, Ltd.

23. Li H, Mihelcic JR, Crittenden JC, Keith A, Anderson (2003) Field Measurements and Modeling of TwoStage Biofilter that Treats Odorous Sulfur Air Emissions. J Environ Eng 129:684-692

24. Lisbon K, McKean M, Shekar S, Svoronos SA, Koopman B (2002) Effect of Dissolved Oxygen on Oxic/Anoxic Diauxic Lag of P.denitrificans. J Environ Eng 128:391-394

25. Monod J (1949) The Growth of Bacterial Cultures.Annu.Rev.Microbiol:371-94

26. Mukherjee AK, Naba K, Bordoloi (2012) Biodegradation of benzene, toluene, and xylene (BTX) in liquid culture and in soil by Bacillus subtilis and Pseudomonas aeruginosa strains and a formulated bacterial consortium. Environmental Science \& Pollution Research 19:3380-3388

27. Padhi S, Kuma, Gokhale S (2017) Treatment of gaseous volatile organic compounds using a rotating biological filter. Bioresour Technol 244:270-280

28. Pala-Ozkok I, Rehman A, Yagci N, Ubay-Cokgor E, Jonas D, Orhon D (2012) Characteristics of mixed microbial culture at different sludge ages: Effect on variable kinetics for substrate utilization. Bioresour Technol 126:274-282

29. Qiu X, Marc A, Deshusses (2017) Performance of a monolith biotrickling filter treating high concentrations of $\mathrm{H}_{2} \mathrm{~S}$ from mimic biogas and elemental sulfur plugging control using pigging. Chemosphere 186:790-797

30. Ras M, Rosa F, Borrull, Rosa Maria M (2008) Determination of volatile organic sulfur compounds in the air at sewage management areas by thermal desorption and gas chromatography-mass spectrometry. Talanta 74:562-9

31. Reardon KF, Douglas C, Mosteller, Julia D, Bull Rogers (2015) Biodegradation kinetics of benzene, toluene, and phenol as single and mixed substrates for Pseudomonas putida F1. Biotechnology \& Bioengineering 69:385-400

32. Strigul N, Dette H, Viatcheslav B, Melas (2009) A practical guide for optimal designs of experiments in the Monod model. Environ Model Softw 24:1019-1026

33. Sun Y, Ming JG, Chen QDongZ, Ye JX, Jian Meng Chen (2016) Characterization of the novel dimethyl sulfide-degrading bacterium Alcaligenes sp. SY1 and its biochemical degradation pathway. J Hazard Mater 304:543-552

34. Wani AH, Anthony K, Lau, Richard MR, Branion (1999) Biofiltration control of pulping odors hydrogen sulfide: performance, macrokinetics and coexistence effects of organo-sulfur species. Journal of Chemical Technology \& Biotechnology 74:9-16

35. Zhang H, Jun JT, Liu YJ, Cao, Yong Tian, Wang (2013) Effects of particle size on lignite reverse flotation kinetics in the presence of sodium chloride. Powder Technol 246:658-663 
36. Zhang X, Yang B, Gao AE, Creamer CC, Cao, Yun Cong Li (2017) Adsorption of VOCs onto engineered carbon materials: A review. J Hazard Mater 338:102-123

37. Zhou Yu, Yang DZ, Chen RY, Zhu, Jian Meng Chen (2011) Substrate interactions during the biodegradation of BTEX and THF mixtures by Pseudomonas oleovorans DT4. Bioresour Technol 102:6644-9

38. Zhou Yu, Yang HL, Huang, Dong Sheng Shen (2016) Multi-substrate biodegradation interaction of 1 , 4-dioxane and BTEX mixtures by Acinetobacter baumannii DD1. Biodegradation 27:1-10

39. Ziagova M, Liakopoulou-Kyriakides M (2007) Kinetics of 2,4-dichlorophenol and 4-Cl- $m$-cresol degradation by Pseudomonas sp. cultures in the presence of glucose. Chemosphere 68:921-927

40. Zwietering MH, Jongenburger I, Rombouts FM and K. Van 'T Riet (1990) Modeling of the bacterial growth curve.Applied \& Environmental Microbiology, 56:1875-81

\section{Figures}
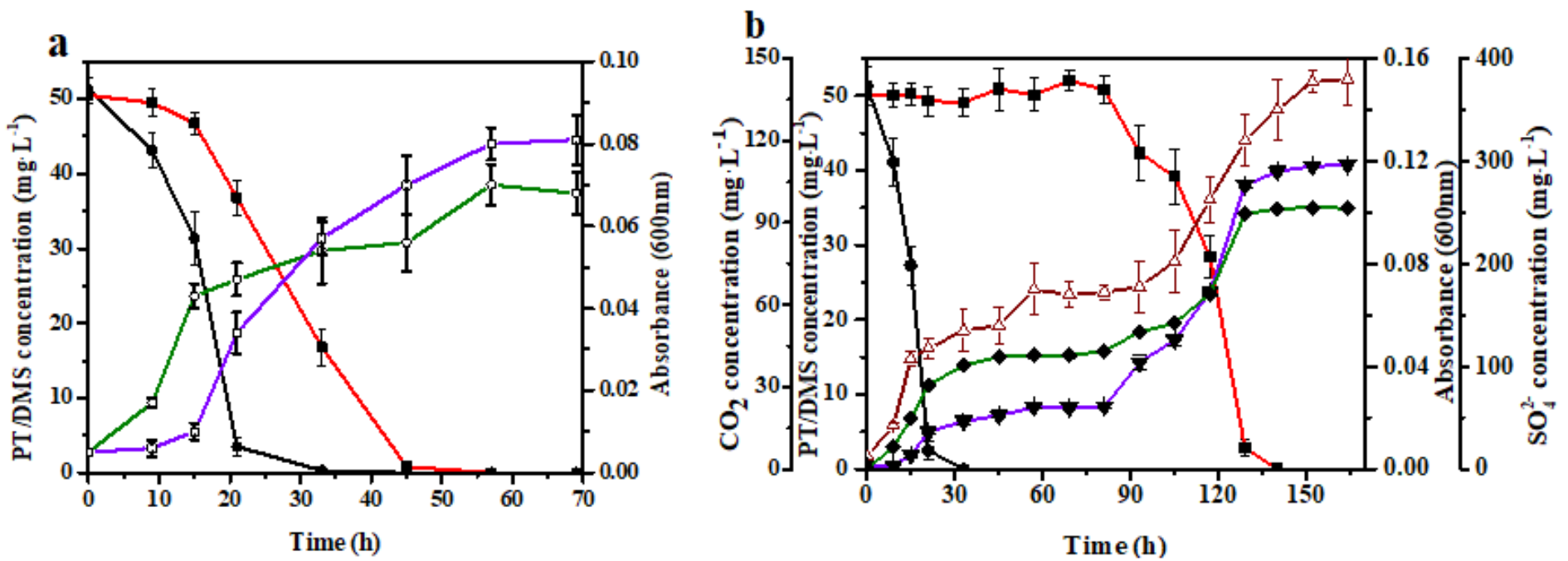

Figure 1

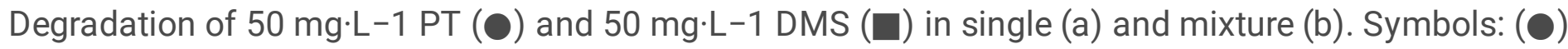
biomass of P. putida S-1 from single PT degradation; $(\square)$ biomass of Alcaligenes sp. SY1 from single

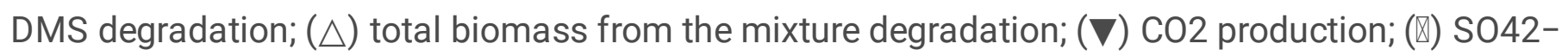
production. 


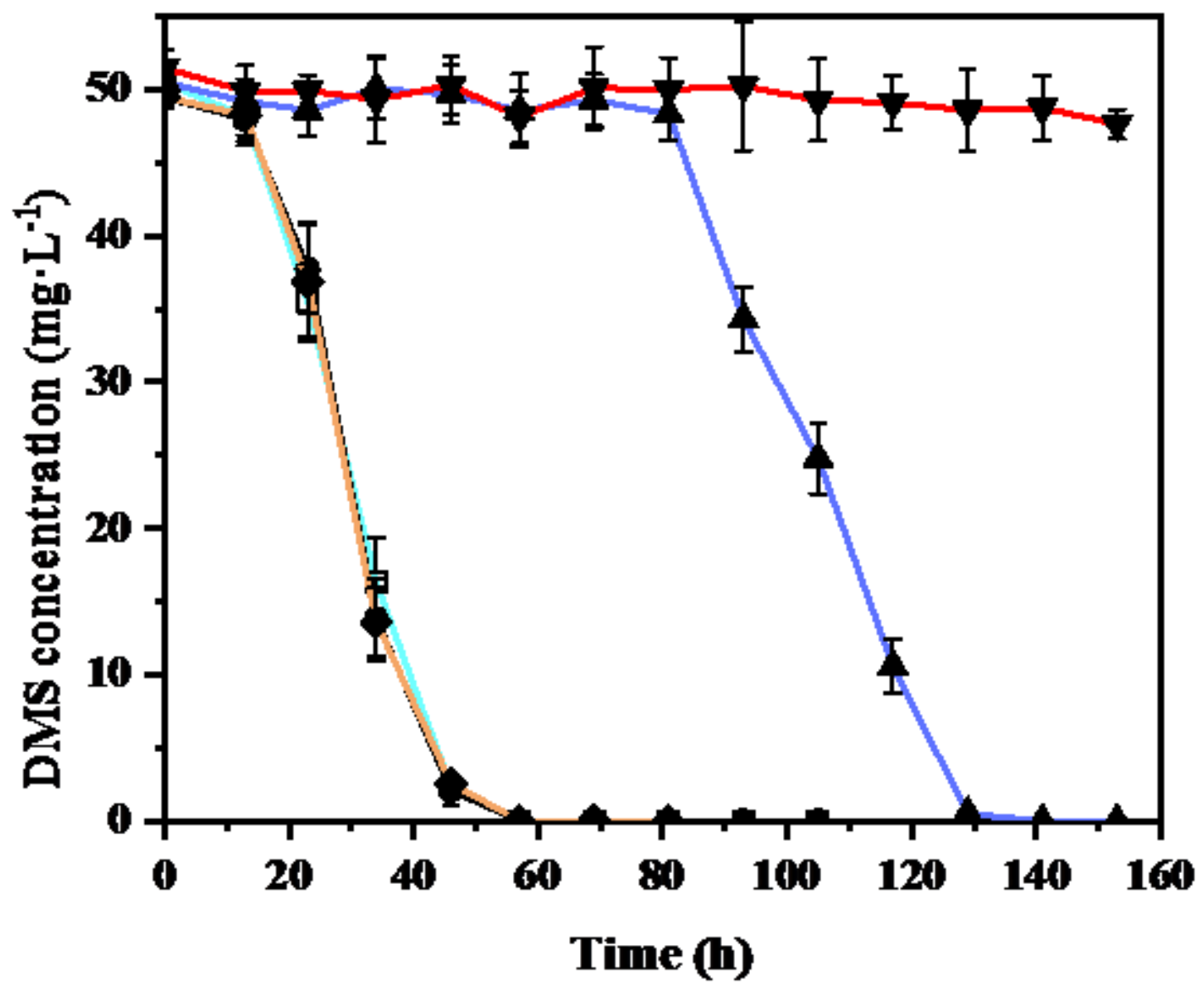

Figure 2

Depletion of DMS under different conditions. Error bars indicate the standard deviation of triplicate samples. Symbols: $(\square)$ Alcaligenes sp. SY1+DMS, ( $(\bullet)$ DMS + Alcaligenes sp. SY1 + P. putida S-1, ( $\boldsymbol{\nabla})$ Alcaligenes sp. SY1+DMS+PT, ( $\mathbf{\Delta})$ DMS+ Alcaligenes sp. SY1+PT+P. putida S-1, (区) DMS and Alcaligenes sp. SY1 were added into the solution where PT had been completely degraded and the biomass of $\mathrm{P}$. putida S-1 had been withdrawn. 


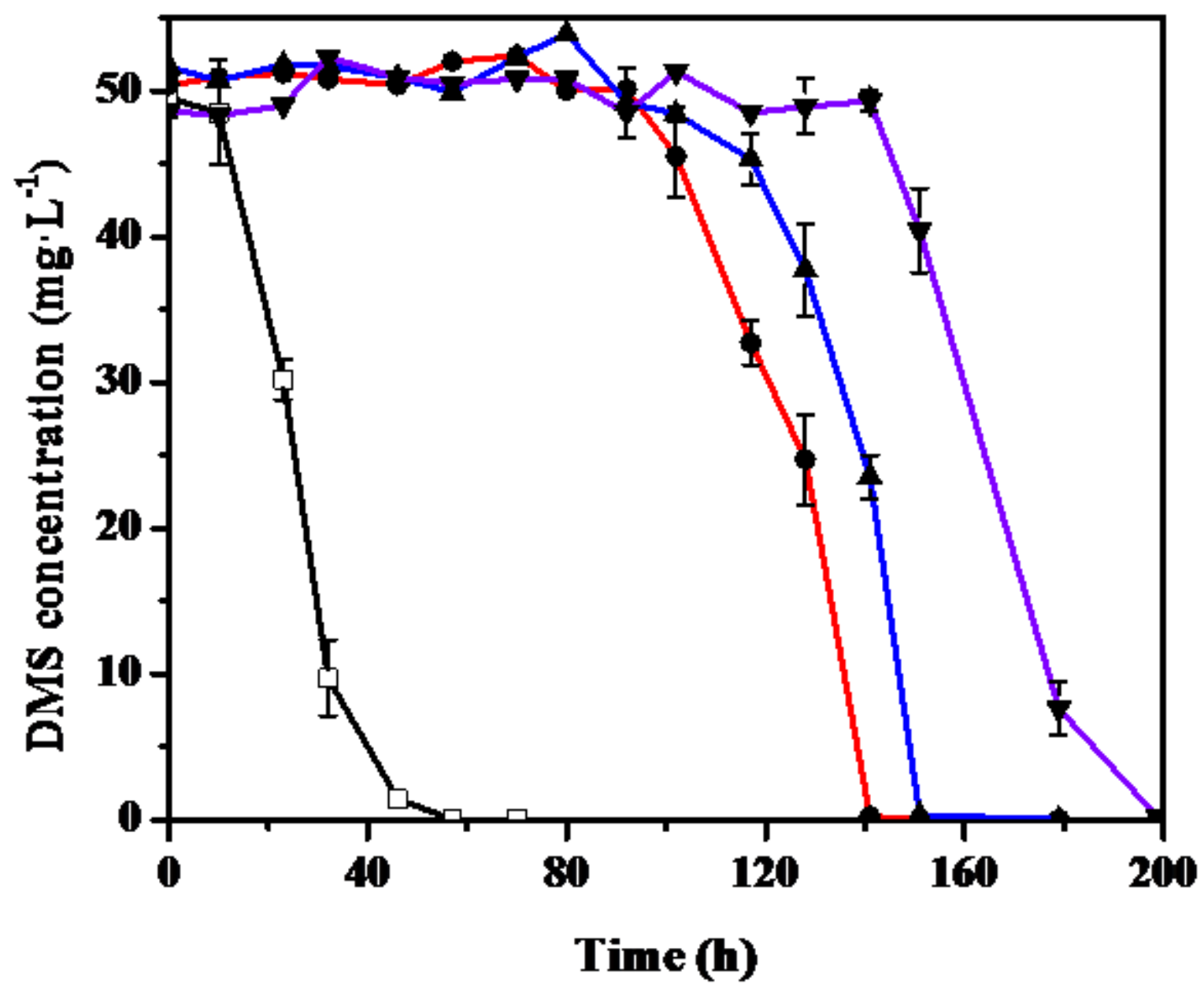

Figure 3

Biodegradation of DMS by Alcaligenes sp. SY1 exposed to $50 \mathrm{mg} \cdot \mathrm{L}-1$ PT for 0 ( $\square), 24(\bullet), 48(\boldsymbol{\Delta})$, and 72 $\mathrm{h}(\boldsymbol{\nabla})$. Error bars indicate the standard deviation of triplicate samples. 

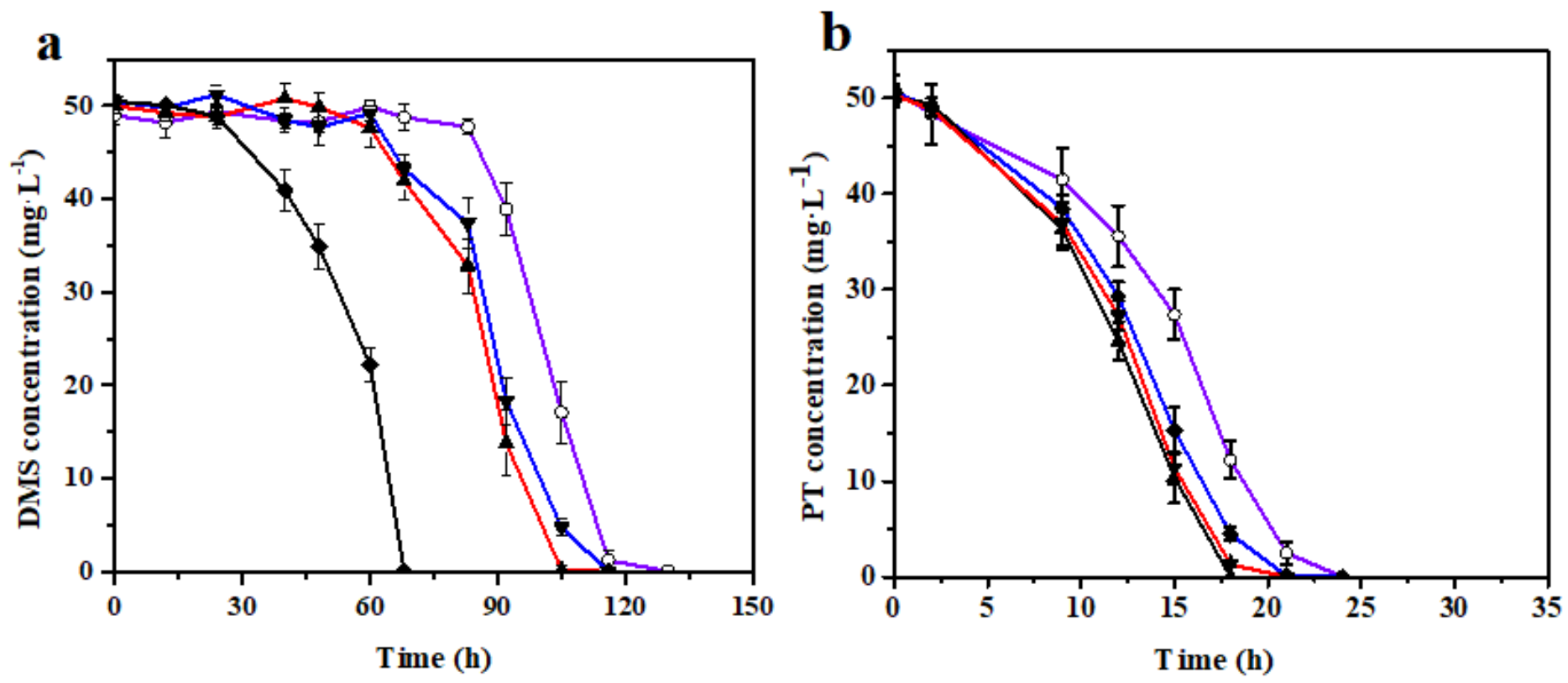

Figure 4

Effect of supplements on DMS biodegradation (a) and PT biodegradation (b) in the dual-substrate mixture. Error bars indicate the standard deviation of triplicate samples. Symbols: (『) citric acid, ( $\mathbf{\Delta})$ yeast extract, $(\boldsymbol{\nabla})$ glucose; $(\mathbf{O})$ control group without supplements.

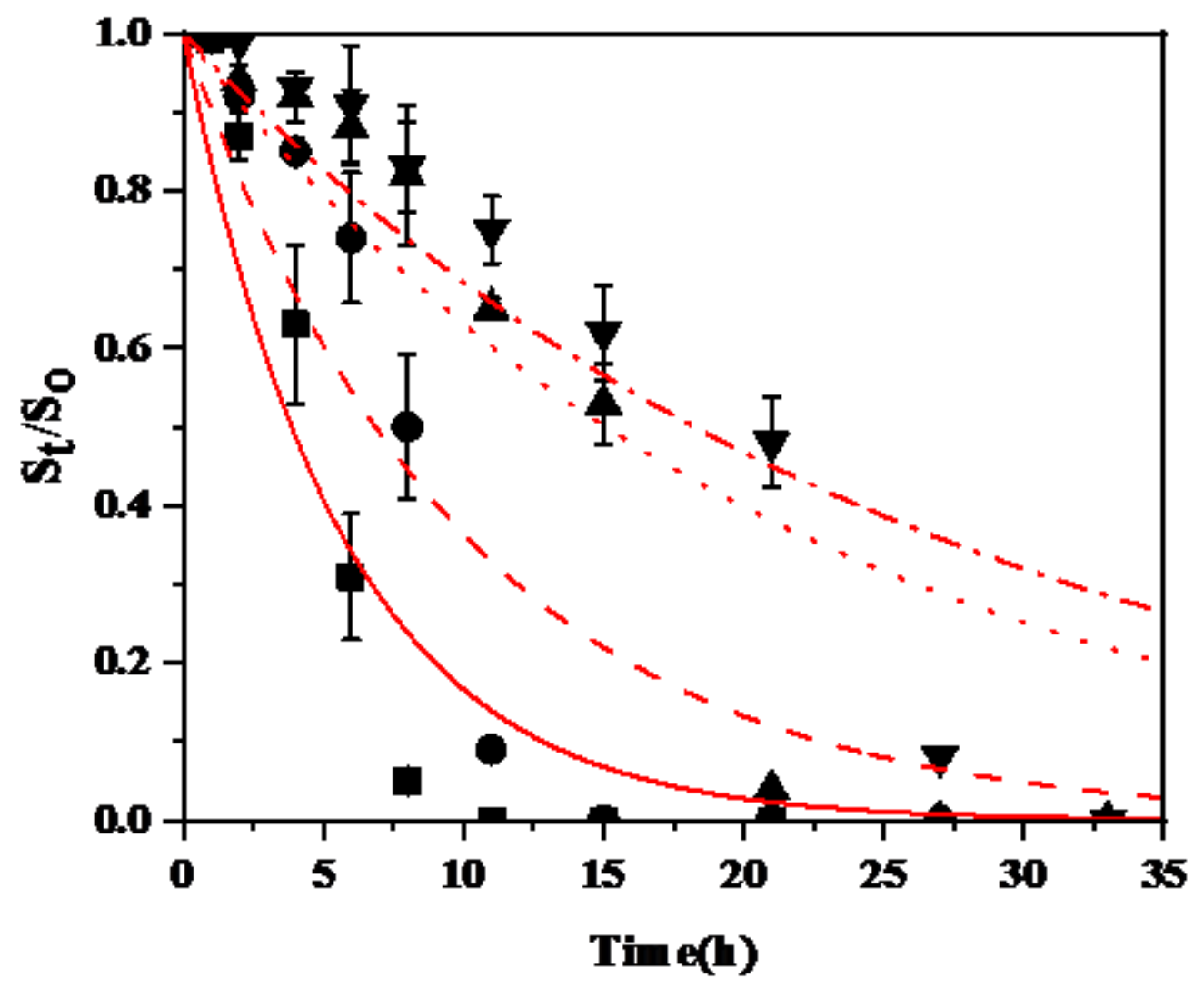


Figure 5

Time course of the relative concentrations (St/So) of $10 \mathrm{mg} \cdot \mathrm{L}-1$ PT ( $\mathbf{\square}-), 20 \mathrm{mg} \cdot \mathrm{L}-1$ PT (--), 50 $\mathrm{mg} \cdot \mathrm{L}-1$ PT $(\boldsymbol{\Lambda} \ldots .$.$) , and 100 \mathrm{mg} \cdot \mathrm{L}-1 \mathrm{PT}\left(\boldsymbol{\nabla}^{-\bullet-)}\right)$ in dual-substrate solution with $50 \mathrm{mg} \cdot \mathrm{L}-1 \mathrm{DMS}$. Symbols represent experimental data, the lines represent first-order kinetic model fitting. Vertical lines represent the standard deviation between duplicate experiments.
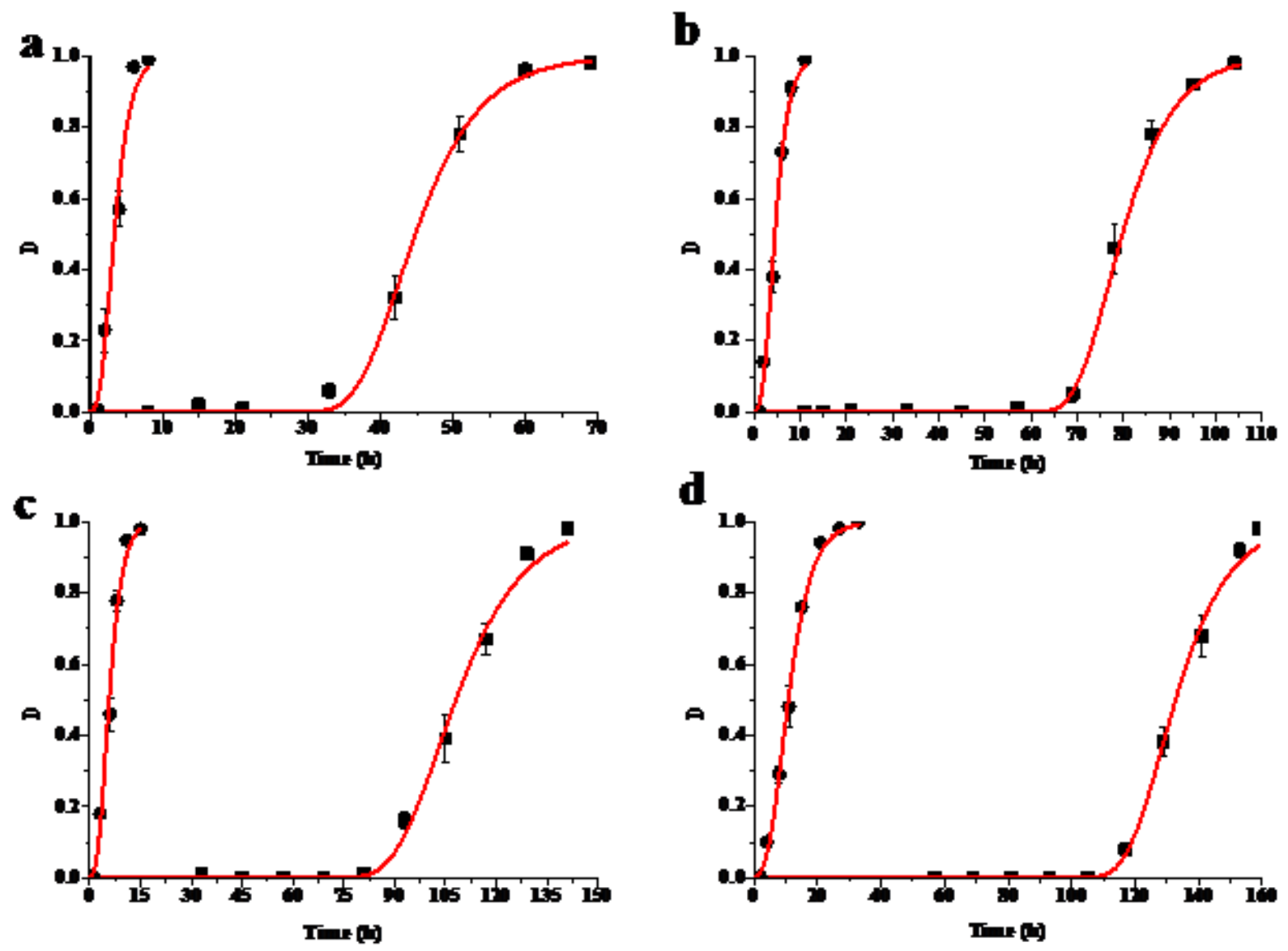

Figure 6

Time course of the cumulative degradation of different concentration combinations of DMS and PT (a: $10 \mathrm{mg} \cdot \mathrm{L}-1$ PT with $50 \mathrm{mg} \cdot \mathrm{L}-1$ DMS; b: $20 \mathrm{mg} \cdot \mathrm{L}-1$ PT with $50 \mathrm{mg} \cdot \mathrm{L}-1$ DMS; c: $50 \mathrm{mg} \cdot \mathrm{L}-1$ PT with 50 $\mathrm{mg} \cdot \mathrm{L}-1$ DMS; d: $100 \mathrm{mg} \cdot \mathrm{L}-1$ PT with $50 \mathrm{mg} \cdot \mathrm{L}-1 \mathrm{DMS})$. Symbols represent experimental data [PT (४), DMS ( $\boldsymbol{\square})$ ], whereas the lines represent Gompertz model fitting. Vertical lines represent the standard deviation between duplicates under the same experimental condition. 